\title{
Apego \& Psicopatología: Una Revisión Actualizada Sobre los Modelos Etiológicos Parentales del Apego Desorganizado
}

\section{Attachment \& Psychopathology: An Update Review of Parental Etiological Models of Disorganized Attachment}

\author{
Felipe Lecannelier \\ Lorena Ascanio \\ Fernanda Flores \\ y \\ Marianela Hoffmann \\ Universidad del Desarrollo, Chile
}

(Rec:2 de junio de 2010; Acept: 2 de abril de 2011)

\begin{abstract}
Resumen
Una de las actuales tendencias dentro de la Teoría del Apego consiste en la comprensión de las trayectorias desviadas en el desarrollo del vínculo, a saber, lo que se conoce como apego desorganizado (apego D). Dos décadas de investigación sobre los antecedentes y consecuentes de este tipo de apego han generado un importante acopio de conocimiento empírico y teórico. Sin embargo, en relación a los modelos etiológicos parentales que buscan explicar los procesos y mecanismos que generan que los niños desarrollen el apego $\mathrm{D}$, la evidencia es contradictoria e incompleta. Dada la enorme relevancia que tiene para la elaboración de estrategias de intervención infantil poseer un conocimiento específico y empíricamente validado sobre los procesos y mecanismos parentales predisponentes al desarrollo de la desorganización del vínculo, es que el presente artículo establece una revisión de los modelos disponibles con miras a aclarar el estado actual del conocimiento en esta temática.
\end{abstract}

Palabras clave: Apego desorganizado, conductas parentales, psicopatología.

\begin{abstract}
One of the actual tendencies within Attachment Theory is about the understanding of atypical trajectories in development of attachment, specifically referred to Disorganized Attachment (D attachment). Two decades of research about antecedents and consequents of this pattern of attachment have spawned a huge amount of empirical and theoretical knowledge. However, parental etiological models who seek to explain processes and mechanisms of D attachment have shown contradictory and incomplete evidence. Due to the important contribution that this kind of models can have for infant and child interventions, this paper seek to review existing model in order to clarify the state of the art of this important research and clinical subject.
\end{abstract}

Key words: Disorganized Attachment, Psychopathology

Correspondencia: Felipe Lecannelier Acevedo, Psicólogo, Centro de Estudios Evolutivos e Intervención en el Niño (CEEIN), Universidad del Desarrollo, Dirección, Santiago. E-mail: flecannelier@udd.cl 


\section{Introducción}

Desde Freud, el tema sobre la influencia de los vínculos tempranos en el desarrollo humano y la salud mental ha sido un tópico central dentro de la Psicología. El Psicoanálisis introdujo una visión subjetiva y clínica muy importante y esclarecedora sobre este tema, con los aportes de Sigmund y Anna Freud, Melanie Klein, Wilfred Bion, Heinz Kohut, Otto Kernberg, y otros. Sin embargo, estos aportes carecieron de dos aspectos esenciales e íntimamente interconectados: por un lado, evidencia empírica/longitudinal sobre esta influencia y por el otro, la realización de análisis prospectivos/evolutivos (más que retrospectivos y clínicos).

La emergencia de la Teoría del Apego y sus estudios empíricos lograron completar este vacío (Bowlby, 1988; Cassidy \& Shaver, 1999, 2008; Lecannelier, 2009). Más de treinta años de estudios empíricos longitudinales han demostrado que si bien los vínculos tempranos con cuidadores significativos tienen una enorme influencia sobre la adaptación psicosocial, ésta influencia no es determinista. En este sentido, el desarrollo es en sí mismo un fluir dinámico de procesos que se van develando en trayectorias normales y/o desviadas afectadas por un sinnúmero de factores que operan en múltiples niveles (Cicchetti \& Cohen, 2006; Grossmann, Grossmann, \& Waters, 2005). Más específicamente, los estudios recientes dentro de la Teoría del Apego se han orientado a investigar las trayectorias desviadas del desarrollo del apego en poblaciones de alto riesgo. Desde esta mirada, el arma de batalla para comprender la psicopatología del apego se ha centrado en lo que se conoce como apego desorganizado (Solomon \& George, 1999). El grado de asociación entre este tipo de apego en edades tempranas y el posterior surgimiento de procesos desadaptativos es de tal magnitud que se ha convertido en una urgencia el conocer de un modo más específico y empírico los antecedentes y consecuentes de este patrón de vinculamiento (Kobak, Cassidy, Lyons-Ruth \& Ziv, 2006).

Los estudios sobre el apego $\mathrm{D}$ pueden dividirse a grandes rasgos en dos tendencias: por un lado están las investigaciones sobre las condiciones y factores que generan el desarrollo de un apego D (lo que se ha llamado en este artículo los modelos etiológicos del apego desorganizado), y por el otro, se encuentran los estudios sobre las consecuencias que este tipo de apego tiene sobre el desarrollo y salud mental de las personas. Esta segunda línea de investigación ha presentado evidencias más claras y consistentes sobre los efectos desadaptivos que el apego desorganizado tiene sobre el desarrollo del niño (para una revisión, ver Green \& Goldwyn, 2002; Lyons-Ruth \& Jacobitz, 2008; y el importante metaanálisis de van Ijzendoorn, Schuengel, \& Bakermans-Kranenburg, 1999). Pero en lo que respecta a las propuestas sobre los modelos etiológicos la evidencia no ha sido consistente en determinar los factores y mecanismos parentales que provocan el desarrollo de un apego D en el niño. Por esta razón se hace relevante revisar y organizar las actuales tendencias y modelos orientados a comprender cuáles son los factores, procesos y mecanismos parentales que hace que los niños desarrollen este patrón de apego, tan perjudicial para su sano desarrollo.

Así mismo, desde la perspectiva de la intervención en salud mental infantil, la imprescindible incorporación de los padres en todo tipo de tratamiento, plantea no solo la urgencia de conocer las causas y explicaciones que hace que éstos desorganicen a sus hijos, sino también la necesidad de crear estrategias de intervención cada vez más específicas y sistemáticas, destinadas al fomento de la seguridad en el apego y la parentalidad positiva.

Para los propósitos de la presente revisión, se utilizarán como criterios de selección de los modelos aquellas propuestas que: en primer lugar, se enmarquen dentro de la Teoría del Apego; en segundo lugar, que hayan sido validadas por a lo menos un estudio empírico (por ejemplo, la propuesta de De Oliveira, Bailey, Moran \& Pederson, (2004) sobre la socialización emocional posee un valor heurístico importante pero no ha sido validada como modelo integrado a nivel empírico). Finalmente, se incluirán aquellos planteamientos que presenten un modelo conceptual organizado bajo la propuesta de explicaciones etiológicas sobre el surgimiento del apego D en los infantes. Este último criterio resulta muy relevante, si se toma como ejemplo un artículo reciente, donde investigadores de la Universidad de Toronto realizaron un estudio que relacionaba los sistemas atencionales automáticos y voluntarios en madres desorganizadas, junto con ciertas implicaciones a nivel cognitivo y neurobiológico, tanto en las madres como en los niños (Atkinson et al., 2009). Estos autores proponen una vía interesante de estudio de los mecanismos etiológicos, pero que todavía no se constituyen en un modelo conceptual organizado.

En consecuencia, el objetivo del presente artículo consiste en revisar los modelos etiológicos que explican la emergencia del apego D con miras a comprender de modo cada vez más específico cuáles son los mecanismos y condiciones parentales que predisponen a dificultades en el desarrollo del apego, y por ende, permitan la elaboración de estrategias de intervención más específicas y basadas en la evidencia.

\section{Historia del Apego Desorganizado.}

La historia sobre la creación y conceptualización (o descubrimiento del apego desorganizado) tiene esa cualidad heurística propia de la ciencia de generar conocimiento nuevo cuando los modelos o propuestas existentes no calzan con nuevas observaciones y evidencias. Ya desde principios de los años setenta, algunos investigadores del apego reportaban que alrededor de un $10 \%$ de casos no podían ser categorizados en los patrones de apego propuestos por Ainsworth y sus colaboradores (Seguro (B), Inseguro 
Evitante (A) e Inseguro Ambivalente (C) (Ainsworth, Blehar, Waters \& Wall, 1978). Este 10\% de infantes desplegaba una serie de conductas en el Procedimiento de la Situación Extraña (PSE) que desafiaba el hecho de que el niño hubiera desarrollado una estrategia coherente para vincularse con la madre, al presentar comportamientos contradictorios, atemorizados, bizarros y claramente conflictivos en el intento de aproximarse al cuidador (Sroufe \& Waters, 1977). Posteriormente, en la década de los ochenta se confirmó esta observación, especialmente en niños que habían sufrido maltrato y que no podían ser clasificados en el modelo A-B-C (Crittenden, 1985; Lyons-Ruth, Connell, Zoll, \& Sthal, 1987). Durante ese mismo periodo, incluso Main \& Weston (1981) encontraron en poblaciones de Nivel Socio-Económico medio, que el 13\% presentaba ese tipo de conductas contradictorias. Con toda esa evidencia reportada, Main \& Solomon (1990), después de re-examinar 200 videos en el PSE, propusieron un listado de conductas que llamaron "desorganizadas/desorientadas", afirmando que el infante desorganizaba su estrategia vincular frente a la madre, expresando conductas que denotaba la pérdida del sentido o meta del apego (búsqueda de protección y regulación del estrés y el peligro). Paralelamente, Main $\&$ Hesse (1990) propusieron un modelo etológico del surgimiento de este fenómeno: para estos investigadores, la aparición del apego D era la expresión de una paradoja evolutiva en donde los padres desplegaban una serie de conductas atemorizadas y/o atemorizantes que dejaban al niño en un estado de "miedo sin solución" al ser los padres la propia fuente de estrés. Esto provocaba en los niños una incapacidad de regular el estrés de la separación y por ende de usar a la madre como una base segura de un modo coherente y organizado.

Por lo tanto, con un instrumento de codificación y una explicación etiológica a mano, en la década de los noventa se observó una explosión de investigaciones destinadas a comprender los antecedentes, consecuentes y desarrollo del apego desorganizado, así como la búsqueda de explicaciones a nivel neurobiológico, genético, psicológico y social (ver van Ijzendoorn, Schuengel, \& Bakermans-Kranenburg, 1999).

A continuación, se revisan aquellos modelos etiológicos parentales, de acuerdo a los criterios de selección anteriormente explicitados.

\section{El Modelo de Main \& Hesse.}

Tal como se mencionó, los principales descubridores del apego D plantearon su modelo etiológico al proponer que los padres, al desplegar conductas que pueden ser atemorizadas $\mathrm{y} / \mathrm{o}$ atemorizantes (ahora en adelante conductas FR) dejan al niño en un estado paradójico de "miedo sin solución" en donde la persona que tiene que calmarlo en situaciones de estrés y peligro es justamente la persona que provoca ese mismo estrés y/o peligro (Hesse \& Main, 2000, 2006; Main
\& Hesse, 1990). En consecuencia, como producto de experimentar este miedo sin solución que inevitablemente implica que el infante no pueda acercarse (ya que el cuidador puede provocarle más estrés) ni alejarse (ya que implicaría que el cuidador lo deja de cuidar) se activan una serie de procesos "donde se contradice o se inhibe la conducta mientras se realiza" (Main \& Hesse, 1990, pp. 173). Estas conductas pueden presentar rasgos relacionados a intenciones contradictorias (acercarse al cuidador con la cabeza baja), miedo (expresiones faciales de miedo), desorientación (vagar perdido por la sala), conductas bizarras (movimientos estereotipados, indirectos, asimétricos, sonidos bizarros, tics, etc.); y conductas de congelamiento.

En lo que respecta a la evidencia, la relación entre estas conductas atemorizadas/atemorizantes (FR) y el apego D ha sido encontrada en tres países diferentes (Abrams, Rifkin, \& Hesse, 2006; Schuengel, Bakermans-Kranenburg, \& van Ijzendoorn, 1999; True, Pisani, \& Oumar, 2001).

¿Pero qué provoca que los padres desplieguen estas conductas FR? Para Main \& Hesse (1990) los padres que presentan un estado mental sobre pérdidas o traumas no resueltos en su historia temprana de apego, evaluado a través de la Entrevista de Apego de Adultos (AAI) predecían un apego D con el hijo. El AAI es una entrevista semiestructurada donde se pide al entrevistado que hable sobre sus emociones sobre sus figuras de apego, que describa eventos específicos de su historia de apego, que detalle eventos traumáticos (abuso o muerte), y que conceptualice cómo esos eventos le han afectado en su vida (Hesse, 1999). La propuesta de estos autores plantea que aquellos padres que desarrollan un apego D en sus hijos, mientras discuten eventos potencialmente traumáticos de sus vidas, muestran signos de desorientación y desorganización a través de lapsos en el monitoreo del discurso (por ejemplo, perder el sentido de la entrevista y de la coherencia del discurso a través de silencios largos, o incapacidad de terminar una frase), y lapsos en el monitoreo del pensamiento (por ejemplo, a través de la explicitación que una persona puede estar muerta y viva a la vez, o negar que un ser querido que está muerto en verdad lo está). Específicamente, estos autores proponen que estos lapsos indican que la persona sigue atemorizada y sobrepasada por sus memorias traumáticas irresueltas, lo que afecta la coherencia del discurso y el pensamiento. Esto a su vez activa conductas de cuidado atemorizadas y/o atemorizantes inexplicables y contradictorias hacia el niño, especialmente en momentos de estrés (Hesse \& Main, 2006). Ejemplos de conductas atemorizantes pueden incluir asustar al niño, tomarlo de un modo muy brusco, abuso físico, sexual, intrusividad extrema, y otras. Ejemplos de conductas atemorizadas pueden incluir entrar en estados de trance frente al niño, buscar cariño y cuidado en el infante, sentir temor hacia él (como qué el niño fuera la fuente de peligro). Específicamente, Hesse \& Main (2006) distinguen los siguientes grupos de conductas 
FR: 1) Amenazantes; 2) Atemorizadas; 3) Disociadas; 4) Tímida; 5) Romántica; 6) Desorganizada.

Por su parte, la evidencia meta-analítica ha demostrado que el $53 \%$ de los padres que tienen este estado irresuelto del apego tienen hijos clasificados como desorganizados en el PSE (van Ijzendoorn, 1995). Esta evidencia ha sido confirmada en Norteamérica, Europa, África y México (Lyons-Ruth \& Jacobitz, 2008).

Por lo tanto, el modelo general de Main \& Hesse se puede graficar de la siguiente manera (Hesse \& Main, 2000; 2006):

Estado mental irresuelto del cuidador con respecto a su propia historia de apego (1) $\otimes$ Conductas atemorizadas/atemorizantes del cuidador hacia su hijo (FR) (2) $\bigotimes$ Desarrollo de un apego desorganizado en el niño (D) (3).

Sin embargo, aunque parece existir evidencia (tal como se ha mencionado) de asociaciones significativas entre 1 y 2 , y 2 y 3 (de un modo separado), no es claro que la conducta FR sea una mediadora entre el estado mental irresuelto y el apego D. Tal como lo plantean algunos autores en la actualidad la evidencia sobre este rol mediador de la conducta parental desorganizadora es escasa y contradictoria (Bernier \& Meins, 2008; Rutter, Kreppner \& Sonuga-Barke, 2009). De los estudios que han intentado probar empíricamente este modelo (es decir, los tres procesos juntos), algunos no pudieron encontrar asociación entre 1 y 2 (Schuengel et al., 1999), y otros no encontraron relación entre 2 y 3 (Lyons-Ruth, Yellin, Melnick, \& Atwood, 2005). Otro estudio realizado en una muestra de bajo riesgo encontró relaciones separadas entre los elementos del modelo, pero no pudo evidenciar que la conducta parental FR fuera un mediador (en cuanto variable explicativa) entre el estado mental irresuelto y el apego D del infante (Goldberg, Benoit, Blokland, \& Madigan, 2003). Por último, un estudio realizado por un importante equipo especializado en estudios de apego D de la Universidad de Western Ontario realizado en una muestra de alto riesgo confirmaron empíricamente el modelo (Madigan, Pederson, \& Moran, 2006).

Por lo tanto, la evidencia apunta al hecho de que la conducta parental FR no puede explicar completamente la relación entre estado mental irresuelto y el apego D en el niño, dejando entrever que pudieran existir otros procesos, factores y mecanismos que expliquen (además de la conducta FR) el surgimiento de un apego D (Madigan, Bakermans-Kranenburg, van Ijzendoorn, Moran, Pederson, \& Benoit, 2006).

\section{El Modelo de Karlen Lyons-Ruth.}

Esta investigadora de la Universidad de Harvard, junto a su equipo se plantean una interesante interrogante sobre la etiología del apego desorganizado: ¿Si la evidencia metaanalítica proveniente de diferentes países revela que el 53\% de los padres con estado mental irresuelto tienen hijos con apego $\mathrm{D}$, entonces qué ocurre con el restante $47 \%$ de padres que no han sufrido pérdidas o traumas no resueltos, pero que desorganizan el apego con sus hijos? Es decir, que esto puede indicar que la propuesta de que el apego $\mathrm{D}$ es producto de estados traumáticos no resueltos puede "constituirse en una ventana muy estrecha para capturar todos los estados mentales anómalos identificados en adultos que tuvieron dificultades en su infancia" (Lyons-Ruth \& Jacobitz, 2008). En este sentido, Lyons-Ruth y colaboradores plantean que las conductas parentales desorganizantes tienen un rango más amplio (que el trauma no resuelto) que pueden incluir formas contradictorias y disruptivas de comunicación afectiva relacionadas a la regulación del estrés y la provisión de cuidado y confort (Lyons-Ruth, Bronfman, \& Atwood, 1999; Lyons-Ruth, Bronfman, \& Parsons, 1999). De esta manera, para que el niño desarrolle un apego $\mathrm{D}$, basta que la conducta parental sea lo suficientemente contradictoria y extrema para que el infante sea incapaz de mantener una estrategia organizada de apego (sea segura, evitante o ambivalente).

Profundizando aún más en su modelo, Lyons-Ruth ha podido describir un patrón clasificatorio de estados mentales propensos a gatillar conductas desorganizantes a través del AAI (lo que llama "estados mentales Hostil-Desamparo" (H-H), así como una serie de criterios conductuales sobre las posibles conductas parentales que desorganizan al niño a través del PSE (que denominó AMBIANCE (Atypical Maternal Behavior Instrument for Assessment and Classification) (Bronfman, Parsons \& Lyons-Ruth, 1993).

En consecuencia, su modelo podría explicarse bajo el siguiente diagrama:

Estados mentales Hostiles-Desamparados en el cuidador $(\mathrm{H}-\mathrm{H})(1) \otimes$ Errores Comunicativos/Afectivos hacia el niño (AMBIANCE) (2) $₫$ apego D en el infante (D) (3).

En el caso de (1), Lyons-Ruth plantea que a través del AAI se puede evaluar el conocido mecanismo de defensa llamado "splitting" en donde la persona puede hacer evaluaciones contradictorias sobre una misma figura de apego, pero separadas en dos sistemas diferentes de memoria (y por ende, accesibles en procesos conscientes paralelos) (Lyons-Ruth, et al., 2005). Ella identifica dos sub-tipos de estados mentales en relación al apego (HH): 1) Sub-tipo Hostil: donde una figura de apego suele generalmente ser representada de una forma negativa (como hostil o malévola), pero suelen aparecer representaciones positivas o que denotan que la persona ha desarrollado una fuerte identificación con el cuidador; 2) Sub-tipo Desamparado: la figura de apego también puede ser evaluada negativamente, pero también co-existen representaciones que evidencian un fuerte lazo con una figura a la que se tuvo que cuidar y proteger. Las aseveraciones negativas no son coherentes ni están integradas con esa actitud cuidadora general.

Los estudios realizados con esta clasificación han mostrado que el sistema $\mathrm{HH}$ es un buen predictor del apego D 
en el niño y presenta una validez discriminante en relación al sistema clásico de codificación del AAI. Así mismo, plantean que la experiencia de un trauma no predice necesariamente apego D si no se consideran los criterios de HH (mostrando que más que el trauma no resuelto en sí mismo, es la presencia de estados mentales disgregados y disociados lo que se relacionaría a este tipo de apego) (Lyons-Ruth et al., 2005). En otro estudio, se encontró que mujeres con Trastorno de Personalidad Limítrofe (TPL) presentaban altos indicadores de $\mathrm{HH}$ comparado con un grupo de mujeres con Distimia, denotando el hecho que la transmisión intergeneracional del apego D suele estar relacionado (además de los traumas no resueltos) a fallas en la integración de estados mentales referentes a figuras significativas de apego (Lyons-Ruth, Melnick, Patrick, \& Hobson, 2007).

Con respecto a los Errores Comunicativos/Afectivos hacia el niño (punto 2), esta investigadora plantea que la desorganización en el infante puede surgir de una multiplicidad de fuentes (no simplemente de estados mentales irresueltos) y por tanto, propone una serie amplia de conductas parentales que poseen el rasgo de ser comunicaciones afectivas contradictorias y disruptivas en relación a la necesidad de ser confortado en el estrés (de ahora en adelante Conductas AMBIANCE) (Lyons-Ruth et al., 1999; Lyons-Ruth et al., 1999). Estas conductas se agrupan en: 1) Errores comunicativos/afectivos (señalamientos contradictorios hacia el infante, fallas en iniciar una respuesta de contención y respuestas inapropiadas en relación a las señales del niño); 2) Confusión de roles y límites (inversión de roles y sexualización); 3) Conducta atemorizada/ desorientada; 4) Intrusividad/negatividad (comunicación física y verbal negativa, atribución negativa, y control con objetos); 5) Evitación (evitación física y verbal, y dirigir lejos con juguetes). Específicamente, bajo este sistema de clasificación se encontraron dos sub-grupos de madres desorganizantes que a su vez se correspondían con dos sub-grupos de infantes desorganizados (Lyons-Ruth \& Spielman, 2004): 1) madres hostiles/autorreferentes: que desplegaban una combinación contradictoria entre ser hostiles e intrusivas por un lado, pero a su vez, buscando constantemente la atención autorreferencial (autocentrada) del infante. Los infantes de estas madres desarrollaban un patrón más de tipo controlador/agresivo con sus madres; 2) madres desamparadas/atemorizadas: que desplegaban una combinación contradictoria entre conductas afectivas por una parte, pero a su vez, evitando al niño de un modo atemorizado y confuso (como si la madre tuviera miedo que el infante pudiera hacerle daño). Los infantes de estas madres mostraban conductas más propias de un patrón controlador/cuidador (tipo inversión de roles).

Recientemente, se han realizado algunos estudios para dar cuenta del modelo de Lyons-Ruth encontrando que las Conductas AMBIANCE se relacionaban significativamente con el apego D. Por ejemplo, Lyons-Ruth et al., (1999) encontraron que el nivel de disrupción comunicativa en la conducta parental y todos los tipos de conductas AMBIANCE predecían significativamente el apego D en los infantes (asociación entre 2 y 3). Esta asociación ha sido replicada en varios estudios provenientes de diferentes países con muestras de madres adolescentes (Madigan et al., 2006), utilizando medidas de mentalización en las madres (Grienenberg, Kelly, \& Slade, 2005), muestras de bajo riesgo (Goldberg et al., 2003), y muestras provenientes de Hungría combinada con medidas genéticas (Gervai et al., 2007).

Sin embargo, tomando el modelo completo de las asociaciones entre 1, 2 y 3, Lyons-Ruth et al., (2005) reportaron que las Conductas AMBIANCE eran un mediador parcial de la influencia entre los estados mentales H-H y la desorganización en el infante, dejando entrever el hecho de que si bien la contribución de la conducta parental en el apego D era un factor importante, existía una porción inexplicada de esta asociación por otros factores, mecanismos y/o procesos. Del mismo modo, el metaanálisis de Madigan et al., (2006) si bien encontró asociaciones entre los tres elementos, los tamaños de efecto fueron bajos.

Por lo tanto, como concluyen Rutter, Kreppner \& Sonuga-Barke (2009): "los orígenes del apego D en las experiencias específicas de cuidado permanecen inciertos" (pp. 531).

\section{El Modelo de Solomon \& George.}

Bowlby propuso que el sistema de apego era un sistema homeostático de regulación de situaciones de estrés y peligro $(1973,1980)$. Esta noción del apego como "sistema de regulación" sigue siendo muy consensuada entre los investigadores (Fonagy \& Target, 1997; Lecannelier, 2002; Schore, 2001; Sroufe, 1996; y otros), implicando que al activarse el sistema de apego, el infante despliega conductas de apego para recuperar su "homeostasis vincular" a través del llanto, la búsqueda, el llamado, etc. buscando ser confortadas, reguladas y protegidas por un cuidador. ¿Pero qué ocurre si el infante no recupera esta homeostasis vincular? Bowlby propuso que estos "asaltos al sistema de apego" generaban estrategias defensivas en el niño bajo la forma de "sistemas segregados" (procesos disociativos). En este sentido, Solomon \& George (1999) tomando esta idea y analizando los datos anómalos de los modelos anteriores, es decir: 1) el hecho de que el apego D se da también en madres de bajo riesgo; 2) que no todas las madres que presentan conductas FR o AMBIANCE tienen niños con apego $\mathrm{D}$, y a su vez, madres de niños con apego seguro podían mostrar alguna de esas conductas, y; 3) el hecho de que no es muy potente la relación entre el estado mental de la madre, su conducta FR o AMBIANCE y el apego $\mathrm{D}$ en el niño) proponen una hipótesis alternativa que llaman "fallas en terminar el sistema de apego" (Solomon 
\& George, 1999; George \& Solomon, 1999). Lo que estos autores proponen es que el rasgo distintivo de la conducta parental desorganizante no necesariamente se relaciona al maltrato, abuso, atemorización, disociación, etc., sino a la incapacidad de reparar el sistema de apego que se ha activado (bajo alguna de las conductas mencionadas anteriormente). Es decir, que más que el error en sí mismo (conductas FR o AMBIANCE), es la falla de la madre en no poder reparar el error. Así lo expresan las autoras: "Lo que puede ser más importante es que las madres de los niños desorganizados fallan en "terminar" el sistema de apego" (Solomon \& George, 1999, pp.14), dejando al niño en un estado crónico de estrés, confusión y daño. Cabe notar, que desde otra línea de investigación Edward Tronick propuso un modelo similar para comprender los errores de coordinación intersubjetiva madre-bebé (Lecannelier, 2006; Gianino $\&$ Tronick, 1988). Por tanto, el efecto desorganizante parece estar dado más por el contexto donde ocurre la conducta y no por la conducta en sí misma. En ese sentido, plantean que muchas de las conductas sutiles propuestas por los modelos FR y AMBIANCE no son lo suficientemente atemorizadoras como para activar en demasía el sistema de apego, pero si la madre falla crónicamente en regular o terminar esta activación, puede dejar al niño en un estado de incapacidad para elaborar estrategias coherentes y predecibles para vincularse con su figura de apego.

Para demostrar empíricamente esta hipótesis, los autores evaluaron las representaciones mentales a través de la narrativa de apego que los infantes desorganizados han desarrollado sobre temas relacionados a la activación del sistema de apego (separación, daño, reunión, etc.) y las representaciones de las madres sobre sus hijos. Este estudio plantea que si las madres no pueden terminar el sistema de apego de sus hijos, dejándolos crónicamente estresados, esto debería expresarse a través de las representaciones mutuas de los miembros de la díada predominando temas que poseen claros índices de desamparo (Solomon \& George, 1996). Los resultados indicaron por un lado, que los niños D y sus madres evidenciaban representaciones mentales cualitativamente diferentes a las madres y niños con apego organizado, y por otro, que se pueden distinguir dos subtipos de desorganización mental: 1) Apego ControladorPunitivo: un primer tipo de niños se representaba el apego bajo eventos caóticos, asustadizos y desintegrados en relación a la separación y la experiencia familiar, como si estos eventos "inundaran" su estado mental (temas de desastres, violencia, explosiones, monstruos, daño, etc.); 2) Apego Controlador-Cuidador: el segundo tipo de niños mostró una tendencia más bien inhibida, evitando pensar en los eventos de apego "como si el niño estuviera tratando de escapar mentalmente de la situación lo más rápido posible" (Solomon \& George, 1999, pp. 17). En el caso de las madres, las investigadoras demostraron que éstas se sienten sobrepasadas e incapaces de regular o "terminar" el estrés de sus hijos, muchas veces evitando cuidarlos. También, encontraron una interesante conexión entre dos tipos de madres asociadas a los tipos de niños mencionados anteriormente: 1) un primer sub-tipo de madres explicitan ser incapaces de manejar y cuidar al niño, quien suele ser descrito como "imposible de manejar", descontrolado y salvaje; 2) el segundo tipo de madres refleja una narrativa que indica una cercanía inusual con el hijo denotando un tipo de inversión de roles donde el niño es representado con capacidades excesivamente autónomas y adultizadas (como un modo de fundamentar que el niño no necesita tanto cuidado, al poseer las competencias para cuidarse por sí mismo). El primer sub-tipo de cuidador corresponde a los niños más bien "inundados" por sus estados mentales, y el segundo tipo de madre a los niños más bien inhibidos.

Los hallazgos de Solomon \& George (1999) sugieren que no es necesario que la conducta de las madre sea tan sutil, atemorizada/atemorizadora, disociada o francamente abusiva tal como lo plantean los modelos anteriores, más bien son instancias que pueden ser comunes y cotidianas donde el sistema de apego ha sido altamente activado (faltando en la provisión de un cuidado y protección adecuado) y la madre no repara ese evento estresante (generando mayor estrés y emocionalidad negativa en el niño). Pero más interesante aún, la evidencia sugiere que estas madres fallan en la terminación del sistema de apego porque ellas mismas experimentan intensas emociones negativas y confusas hacia el niño, sea bajo la forma de "inundación" de estados negativos o inhibición de estos estados. En este sentido, las madres de los niños controladores-cuidadores sienten una necesidad de inhibir su conducta de cuidado, alejándose del niño y/o psicológicamente abandonándolo (dejándolo en un estado crónico de desamparo y miedo). Esto a su vez obliga al niño a inhibir sus estados mentales para estar cerca de su madre. Por otra parte, las madres de los niños controladores-punitivos se descontrolan e inundan de sus afectos negativos hacia el niño (gritándoles, pegándoles, molestándolos, etc.), quienes tienden a usar también la rabia y la punición para controlar y llamar la atención de sus cuidadores.

\section{El modelo de Koós \& Gergely.}

Basado en la teoría del Biofeedback Social (Gergely \& Watson, 1996, 1999; y en español, ver Lecannelier, 2009), Koós \& Gergely (2001) proponen un modelo etiológico del apego D. Numerosos estudios han demostrado que bebés de incluso 2 meses suelen detectar (y alegrarse!) cuando sus acciones son contingentes a estímulos externos (es decir, que el bebé tiene in control causal sobre esos estímulos, por ejemplo al mover su pie y que eso produzca un movimiento en un móvil) (Watson, 1994). Incluso, los bebés pueden detectar diferentes grados de contingencia entre su conducta y el estímulo a través de lo que Gergely \& Watson, (1999), llaman un "modulo innato de detección 
de contingencias". Se ha postulado que una contingencia perfecta le permite al bebé distinguir una experiencia proveniente de sí mismo, mientras que una contingencia alta pero no perfecta que vendría de "fuera" del bebé, tales como las acciones de los cuidadores, le permiten distinguir experiencia de no sí-mismo (Watson, 1994). La evidencia plantea que durante los primeros 3 meses de vida el bebé prefiere la contingencia perfecta (quizás como un modo de explorar y distinguir experiencias de sí-mismo), pero posterior a los 3 meses prefiere contingencias altas pero imperfectas, como un modo de orientarse y explorar el mundo social. Pero para que este desarrollo siga su curso normativo, el mundo social (los cuidadores) tiene que ser lo suficientemente predecible y controlable para proveer de estas contingencias altas pero imperfectas, y así desarrollar un sentido de eficacia y seguridad emocional en el apego del bebé. ¿Pero qué ocurre entonces en el caso de padres que despliegan una serie de conductas impredecibles, incontrolables, confusas, atemorizadas/atemorizantes, abusivas, etc.? Es decir, lo que Koós \& Gergely (2001) llaman un "ambiente de contingencia desviado". Ellos proponen la "hipótesis del cambio parpadeante (flickering switch)" estableciendo que existen periodos en donde los padres desorganizantes no realizan conductas FR o AMBIANCE o de no "terminar el apego" y en esos momentos el bebé puede hacer la transición normativa desde contingencias perfectas (detección del sí-mismo) a contingencias altas pero imperfectas (detección del mundo social). En otros momentos, cuando los padres exhiben estas conductas, el infante pierde el control contingente, experimentando ansiedad y desamparo activando un "cambio (defensivo) parpadeante" hacia la búsqueda del modo original perfectamente contingente. Como resultado de esto, el sistema atencional se desconecta del mundo social (los cuidadores) y se hiperconecta con el sí-mismo, volviéndose esto un modo predominante para el bebé, y derivando en un estilo disociado de organización atencional (ya que el bebé tiene que orientar su atención hacia lo social, pero busca constantemente hacerlo hacia el sí-mismo). Este switch parpadeante hacia la contingencia perfecta puede explicar porqué muchos niños con apego D hacen conductas autorreguladoras extremas (conductas repetitivas y estereotipadas tales como cabeceo, tirarse las orejas, el pelo, la piel, etc.) ya que el niño está disociadamente buscando la experiencia de contingencia hacia sí mismo, y desconectándose de la contingencia social. En un estudio realizado con 60 díadas evaluadas a los 6.5 meses (medidas de contingencia emocional) y posteriormente a los 12 meses (PSE-estilo de apego) se encontró que los infantes $\mathrm{D}$ (a diferencia de los otros patrones de apego) tienden a buscar preferentemente fuentes de estimulación de contingencia perfecta, posterior a una conducta de "cara inmóvil de la madre, y eso les generaba afecto positivo.

Los aportes de la Teoría del BioFeedback Social son muy interesante para la psicopatología (ver Fonagy, Gergely,
Jurist, \& Target, 2002). Sin embargo, el modelo propuesto puede explicar el tipo de conductas autorreguladoras extremas, pero necesita más desarrollo y especificación para comprender todo el proceso de desorganización vincular, y sus consecuencias a nivel de la psicopatología.

\section{El modelo de Bernier \& Meins.}

Como un modo de resolver las inconsistencias y discrepancias sobre los procesos etiológicos que pueden predecir el desarrollo de un apego D, Annie Bernier \& Elizabeth Meins recientemente propusieron su "modelo de umbral" (Bernier \& Meins, 2008). Considerando toda la evidencia disponible, estas autoras proponen que la desorganización opera bajo un sistema de umbral que hace al infante más o menos vulnerable o resiliente para desarrollar un apego desorganizado. Los parámetros de este umbral están dados por: 1) Características del niño: esto incluye ciertas predisposiciones genéticas que al interactuar con ambientes adversos pueden generar una mayor predisposición al apego D. Al respecto, los últimos años han presenciado un intenso debate sobre si el polimorfismo genético del gen receptor de la dopamina (DRD4) en interacción con ciertas dificultades parentales (tales como estados mentales no resuelto) puede ser un factor de riesgo para la desorganización (Gervai et al., 2007; Lakatos, Toth, Nemoda, Ney, Sasvari-Szekely \& Gervai, 2002; van Ijzendoorn \& Bakermans-Kranenburg, 2006). Sin embargo, cabe destacar que la mayoría de estos estudios consideran la vulnerabilidad genética del niño, pero no de la madre (a excepción del estudio de Gervai et al., 2007, pero no encontrando relación con su conducta AMBIANCE, ni con el apego D del infante). La otra fuente de características del niño proviene de los procesos temperamentales, en donde ciertas predisposiciones heredables (tales como la irritabilidad e hiperreactividad) pueden propender a cambios en las conductas de cuidado del niño (para una revisión, ver Vaughn \& Bost, 1999). Por último, una línea importante de estudios ha demostrado ciertas vulnerabilidades a nivel psicofisiológico y psiconeuroinmunologico que pueden afectar la organización del apego (Spangler \& Grossmann, 1999).

2) Nivel de sensibilidad y determinadas características parentales; incluyen el estado mental de los padres con respecto al apego (variable ya mencionada en los modelos anteriores) al afectar o bajar el umbral para realizar conductas atípicas propensas a desorganizar el apego en un niño; el nivel de sensibilidad parental (aunque solo suele provocar apego D en combinación con factores de riesgo contextuales, tales como el NSE); la capacidad de empatía o mentalización de los padres (Fonagy, Gergely, \& Target, 2007; Meins, Fernyhough, Fradley \& Tuckey, 2001); y la presencia de otros cuidadores (Howes, Rodning, Galluzo, \& Myers, 1988); 3) como tercer parámetro describen los factores de riesgo socio-ambientales: es bien sabido que los estresores socio-económicos acumulados afectan el nivel 
de sensibilidad de los padres y determinados procesos en el niño, y por ende, pueden propender a desorganizar al infante. La evidencia ha demostrado que las disputas maritales, embarazo adolescente, abuso de sustancia y el estrés parentales se relacionan al apego D (Kobak et al., 2006; van Ijzendoorn et al., 1999).

En consecuencia, bajo la operación de la "fórmula" de estas tres variables cada niño puede estar en un punto del umbral que lo puede dejar más o menos propenso a desarrollar apego D. Por ejemplo, un niño puede vivir con padres con alta insensibilidad, pero tiene ciertas características genéticas y temperamentales que le permiten organizar mejor su apego, y por ende, protegerlo de desorganizarse en el vinculo. Lo mismo se puede aplicar combinando los tres set de variables anteriormente mencionados. Dos comentarios se pueden hacer en relación a esta propuesta. Por un lado, entrega una visión más realista sobre la etiología del apego desorganizado al considerar diferentes procesos que operan en múltiples niveles (más acorde con los principios de la psicopatología del desarrollo que permiten analizar las diferencias individuales de cada niño, su familia y su contexto). Por otra parte, deja sin explicar de un modo delimitado cuáles serían los mecanismos parentales que desorganizan a un niño, y la posibilidad de elaborar estrategias terapéuticas más específicas y sistemáticas para este tipo de niños en alta condición de riesgo y vulnerabilidad.

\section{Comentarios finales}

Ciertas conclusiones y reflexiones son importantes a articular, de acuerdo a lo anteriormente revisado: en primer lugar, aclarar que el apego desorganizado no es una patología en sí misma, sino más bien podría ser considerado un proceso que dificulta la organización de la experiencia psicofisiológica, emocional, cognitiva y relacional predisponiendo a la persona a experimentar mayores dificultades para regular las situaciones estresantes (y/o traumáticas) propias de la vida. Recientes evidencias apuntan a que la desorganización parental equipa al niño para usar la disociación como mecanismo de regulación de estados mentales, dejando al infante en estado de vulnerabilidad para manejar el estrés en futuras ocasiones (Carlson, 1998; Hesse \& Main, 2006; Liotti, 1999); en segundo lugar, la evidencia revisada en el presente documento establece que si bien los padres (o cuidadores principales) son los principales generadores de desorganización en el niño, plantear modelos parentales unívocos resulta un grave error en la actualidad. Es decir, que la propuesta de modelos que planteen que es un solo proceso (por ejemplo, el estado mental irresuelto del apego) o un solo mecanismo (por ejemplo, sistemas atencionales disgregados), o un solo nivel de funcionamiento (vulnerabilidad genética) suelen caer en el error reduccionista que inevitablemente deja fuera la complejidad del proceso de la parentalidad. Por otra parte, el planteamiento de modelos generalistas multinivel y multiproceso (tal como el modelo de umbral, o el conocido modelo de Bronfrenbrenner) proveen un marco general esclarecedor para iniciar la comprensión del objeto a estudiar, pero deja sin explicar el fenómeno a investigar al proponer fundamentos "all too nothing" (demasiados abarcativos) (Rutter, 1997) (entiendo por explicación "premisas que involucran algún mecanismo (Bunge, 2001). En consecuencia, un posible dilema a resolver (y no solo en el caso de las explicaciones etiológicas parentales predisponentes a la desorganización) sería la propuesta de modelos que por un lado abarquen un número importante de multiprocesos y multinivel, y que a su vez conserven el estatus de explicación. Ambas condiciones, será lo que al final permitirá generar una intervención específica para modificar/desarrollar/aumentar el mecanismo propuesto (cualquiera que sea).

\section{Referencias}

Abrams, K., Rifkin, A., \& Hesse, E. (2006). Examining the role of parental frightened/frightening subtypes in predicting disorganized attachment within a brief observational procedure. Development and Psychopathology, 18, 344-362.

Ainsworth, M.D.S., Blehar, M.C., Waters, E., \& Wall, S. (1978). Patterns of Attachment: A Psychological Study of the Strange Situation. Hillsdale, NJ: Erlbaum.

Atkinson, L., Leung, E., Goldberg, S., Benoit, D., Poulton, L., Myhal, N., Blokland, K., \& Kerr, Sh. (2009). Attachment and selective attention: Disorganization and emotional stroop reaction time. Development \& Psychopathology, 21, 99-126.

Bernier, A., \& Meins, E. (2008). A threshold approach to understanding the origins of attachment disorganization. Developmental Psychology, 4, 969-982.

Bowlby, J. (1973). Attachment and loss: Vol.2. Separation. New York: Basic Books.

Bowlby, J.(1980). Attachment and loss: Vol.3. Loss. New York: Basic Books.

Bowlby, J. (1988). Una base segura. Aplicaciones clínicas de una teoría del apego. Buenos Aires: Paidós.

Bronfman, E., Parsons, E., \& Lyons-Ruth, K. (1993). Atypical Maternal Behavior Instrument for Assessment and Clasification (AMBIANCE): Manual for coding disrupted affective communication. Unpublished manual. Department of Psychiatry, Cambridge Hospital.

Bunge, M. (2001). Crisis y reconstrucción de la filosofía. Madrid. Gedisa Editorial.

Carlson, E. (1998). A prospective longitudinal study disorganization/ disorientation. Child Development, 69, 1107-1128.

Cassidy, J., \& Shaver, Ph. (Eds.) (1999). Handbook of Attachment. Theory, Research and Clinical Applications. $1^{\text {st }}$ Edition. New York: Guilford Press.

Cassidy, J., \& Shaver, Ph. (Eds.) (2008). Handbook of Attachment. Theory, Research and Clinical Applications. $2^{\text {nd }}$ Edition. New York: Guilford Press.

Cicchetti, D., \& Cohen, D. (Eds). (2006). Developmental psychopathology. Theory and method ( $2^{\text {nd }}$ ed.). New York: Wiley.

Crittenden, P.M. (1985). Social networks, quality of parenting, and child development. Child Development, 56, 1299-1313.

De Oliveira, C.A., Bailey, H.N., Moran, G., \& Pederson, D.R. (2004). Emotion socialization as a framework for understanding the development of disorganized attachment. Social Development, 13, 437-467.

Fonagy, P., \& Target, M. (1997). Attachment and reflective function: Their role in self-organization. Development and Psychopathology, 9, 679-700. 
Fonagy, P., Target, M., \& Gergely, G. (2000). Attachment and borderline personality disorder. The Psychiatric Clinics of North America, 23, 103-122.

Fonagy, P., Gergely, G., \& Target, M. (2007). The parent-infant dyad and the construction of the subjective self. Journal of Child Psychology and Psychiatry, 48, 288-328.

Fonagy, P., Gergely, G., Jurist, E.L., \& Target, M. (2002). Affect regulation, mentalization and the development of the self. New York: Other Press.

George, C., \& Solomon, J. (1999). Attachment and caregiving: The caregiving behavioral system. En J. Cassidy \& P.R. Shaver (Eds), Handbook of attachment: Theory, research, and clinical applications (pp. 649-670). New York: Guilford Press.

Gergely, G., Watson, J.S. (1996), The social biofeedback theory of parental affect-mirroring. International Journal of Psycho-Analysis, 77, 1181-1212.

Gergely, G., \& Watson, J. (1999). Early social-emotional development: Contingency perception and the social biofeedback model. En P. Rochat (Ed), Early social cognition: Understanding others in the first months of life (pp.101-137). Hillsdale, NJ: Erlbaum.

Gervai, J., Novak, A., Lakatos, K., Toth, I., Danis, I., Ronai, Z., et al., (2007). Infant genotype may moderate sensitivity to maternal affective communications: Attachment disorganization, quality of care, and the DRD4 polymorphism. Social Neuroscience, 2, 1-13.

Gianino, A., \& Tronick, E.Z. (1988). The mutual regulation model: The infant's self and interactive regulation, coping and defense. En T. Field, P. McCabe, \& N. Schneiderman (Eds.), Stress and coping. Hillsdale, NJ: Erlbaum.

Goldberg, S., Benoit, D., Blokland, K., \& Madigan, S. (2003). Atypical maternal behavior, maternal representations, and infant disorganized attachment. Development and Psychopathology, 15, 239-257.

Green, J., \& Goldwyn, R. (2002). Annotation: Attachment disorganization and psychopathology: New findings in attachment research and their potential implications for developmental psychopathology in childhood. Journal of Child Psychology and Psychiatry, 43, 835-846.

Grienenberger, J.F., Kelly, K., \& Slade, A. (2005). Maternal reflective functioning, mother-infant affective communication, and infant attachment: Exploring the link between mental states and observed caregiving behavior in the intergenerational transmission of attachment. Attachment and Human Development, 7, 299-311.

Grossmann, K., Grossmann, K.E., \& Waters, E. (2005). Attachment from infancy to adulthood: The major longitudinal studies. New York: Guilford Press.

Hesse, E. (1999). The Adult Attachment Interview: Historial and Current Perspectives. En S. Cassidy \& Ph. Shaver (Eds.), Handbook of Attachment. Theory, Research and Clinical Applications (pp.395-433). New York. Guilford Press.

Hesse, E., \& Main, M. (2000). Disorganized infant, child and adult attachment: Collapse in behavioral and attentional strategies. Journal of the American Psychoanalytic Association, 48, 1117-1127.

Hesse, E., \& Main, M. (2006). Frightened, threatening, and dissociative parental behavior: Theory and associations with parental adult attachment interview and infant disorganization. Development \& Psychopathology, 18, 309-343.

Howes, C., Rodning, C., Galluzo, D.C., \& Myers, L. (1988). Attachment and child care: Relationship with mother and caregiver [Special Issue on infant day care]. Early Childhood Research Quarterly, 36, 403-441.

Kobak, R., Cassidy, J., Lyons-Ruth, K., \& Ziv, Y. (2006). Attachment, stress, and psychopathology: A developmental pathways model. En D. Cicchetti \& D.J. Cohen (Eds), Handbook of developmental psychopathology. Theory and method (pp. 333-369). $2^{\text {nd }}$ ed. New York: Wiley \& Sons.

Koós, O., \& Gergely, G. (2001). A contingency-based approach to the etiology of "disorganized" attachment: The "flickering switch" hypothesis. En J. Allen (Ed), Cognitive and interactional foundations of attachment, Special issue of the Bulletin of the Menninger Clinic, 65, 397-410.

Lakatos, K., Toth, I., Nemoda, Z., Ney, K., Sasvari-Szekely, M., \& Gervai, J. (2002). Further evidence of the role of Dopamine D4 receptor
(DRD4) gene in attachment disorganization: Interaction of the exon III $48 \mathrm{bp}$ and the $-521 \mathrm{C} / \mathrm{T}$ promoter polymorphisms. Molecular Psychiatry, 7, 27-31.

Lecannelier, F. (2002). El legado de los vínculos temprano: Apego y autorregulación. Revista Chilena de Psicoanálisis, 19, 191-201.

Lecannelier, F. (2006). Apego e intersubjetividad. Influencia de los vínculos tempranos en el desarrollo humano y la salud mental PARTE I. Santiago: LOM.

Lecannelier, F. (2009). Apego e intersubjetividad. Influencia de los vínculos tempranos en el desarrollo humano y la salud mental PARTE II. La Teoría del Apego. Santiago: LOM.

Liotti, G. (1999). Disorganization of attachment as a model for understanding dissociative psychopathology. En J. Solomon \& C. George (Eds), Attachment Disorganization (pp. 291-317). New York: Guilford Press.

Lyons-Ruth, K., Bronfman, E., \& Atwood, G. (1999). A relational diathesis model of hostile-helpless states of mind: Expressions in mother-infant interaction. En J. Solomon \& C. George (Eds.), Attachment Disorganization (pp. 33-70). New York: Guilford Press.

Lyons-Ruth, K., Bronfman, E., \& Parsons, E. (1999). Atypical maternal behaviour and disorganized infant attachment strategies: Frightened, frightening, and atypical maternal behaviour and disorganized infant attachment strategies. En J. Vondra \& D. Barnett (Eds), Atypical patterns of infant attachment: Theory, research, and current directions. Monographs of the Society for Research in Child Development, 643, Serial No. 258).

Lyons-Ruth, K., Connell, D., Zoll, D., \& Stahl, J. (1987). Infants at social risk: Relations among infant maltreatment, maternal behavior, and infant attachment behavior. Developmental Psychology, 23, 223-232.

Lyons-Ruth, K., \& Jacobitz, D. (1999). Attachment disorganization. Genetic factors, parenting contexts, and developmental transformation from infancy and adulthood. En J. Cassidy, J \& Ph. Shaver (Eds.), Handbook of Attachment. Theory, Research and Clinical Applications (pp.666-697). $2^{\text {nd }}$. New York: Guilford Press.

Lyons-Ruth, K., Melnick., S., Patrick, M., \& Hobson, R.P. (2007). A controlled study of hostile-helpless states of mind among borderline and dysthymic women. Attachment and Human Development, 9, 1-6.

Lyons-Ruth, K., \& Spielman, E. (2004). Disorganized infant attachment strategies and helpless-fearful profiles of parenting: Integrating attachment research with clinical intervention. Infant Mental Health Journal, $25,318-335$.

Lyons-Ruth, K., Yellin, C., Melnick, S., \& Atwood, G. (2005). Expanding the concept of unresolved mental states: Hostile/hepless states of mind on the Adult Attachment Interview are associated with disrupted mother-infant communication and infant disorganization. Development and Psychopathology, 27, 1-23.

Madigan, S., Bakermans-Kranenburg, M.J., van Ijzendoorn, M.H., Moran, G., Pederson, D., \& Benoit, D. (2006). Unresolved states of mind, anomalous parental behavior, and disorganized attachment: A review and meta-analysis of a transmission gap. Attachment and Human Development, $8,89-111$.

Madigan, S., Pederson, D., \& Moran, G. (2006). Unresolved states of mind, disorganized attachment relationships, and disrupted mother-infant interactions of adolescent mothers and their infants. Developmental Psychology, 42, 293-304.

Main, M., \& Weston, D.R. (1981). The quality of the toddler's relationship to mother and to father: Related to conflict behaviour and the readiness to establish new relationships. Child Development, 52, 932-940.

Main, M., \& Solomon, J. (1990). Procedures for identifying infants as disorganized/disoriented during the Ainsworth Strange Situation. En M. Greenberg, D. Cicchetti, \& E.M. Cummings (Eds.), Attachment in the preschool years: Theory, research and intervention (pp.121-160). Chicago: University of Chicago Press.

Main, M., \& Hesse, E. (1990). Parents' unresolved traumatic experiences are related to infant disorganized attachment status: Is frightened and/or frightening parental behavior the linking mechanism? En M. Greenberg, D. Cicchetti, \& E.M. Cummings (Eds.), Attachment in the preschool 
years: Theory, research and intervention (pp.161-184). Chicago: University of Chicago Press.

Meins, E., Fernyhough, C., Fradley, E., \& Tuckey, M. (2001). Rethinking maternal sensitivity: Mothers` comments on infants` mental processes predict security of attachment at 12 months. Journal of Child Psychology and Psychiatry, 42, 637-648.

Rutter, M. (1997). Clinical implications of attachment concepts: Retrospect and prospect. En L. Atkinson \& K.J. Zucker (Eds.), Attachment and psychopathology (pp. 17-46). New York: Guilford Press.

Rutter, M., Kreppner, J., \& Sonuga-Barke, E. (2009). Emanuel Miller Lecture: Attachment insecurity, disinhibited attachment, and attachment disorders: Where do research findings leave the concepts? Journal of Child Psychology and Psychiatry, 50, 529-543.

Schore, A.N. (2001a). Effects of a secure attachment relationship on right brain development, affect regulation, and infant mental health. Infant Mental Health Journal, 22, 7-66.

Schuengel, C., Bakermans-Kranenburg, M.J., \& van Ijzendoorn, M.H. (1999). Frightening maternal behaviour linking unresolved loss and disorganized infant attachment. Journal of Consulting and Clinical Psychology, 67, 54-63.

Solomon, J., \& George, C, (1996). Defining the caregiving system: Toward a theory of caregiving. Infant Mental Health Journal, 17, 183-197.

Solomon, J., \& George, C. (1999). Attachment disorganization. New York: Guilford Press.

Solomon, J., \& George, C. (1999). The place of disorganization in attachment theory. Linking classic observations with contemporary findings. En J. Solomon \& C. George (Eds.), Attachment Disorganization (pp. 3-32). New York: Guilford Press.

Spangler, G., \& Grossmann, K. (1999). Individual and physiological correlates of attachment disorganization in infancy. En J. Solomon \& C. George (Eds.), Attachment Disorganization (pp. 95-126). New York: Guilford Press.
Sroufe, A.L. (1996). Emotional development. The organization of emotional life in the early years. New York: Cambridge University Press.

Sroufe, A.L., \& Waters, E. (1977). Attachment as an organizational construct. Child Development, 48, 1184-1199.

True, M., Pasani, L., \& Oumar, F. (2001). Infant-mother interactions among the Dogon of Mali. Child Development, 72, 1451-1466.

Vaughn, B.E., \& Bost, K.K. (1999). Attachment and temperament: Redundant, independent, and interacting influences on interpersonal adaptation and personality development. En S. Cassidy \& Ph. Shaver (Eds.), Handbook of Attachment. Theory, Research and Clinical Applications (pp.198-225). New York. Guilford Press.

van Ijzendoorn, M.H. (1995). Adult attachment representations, parental responsiveness, and infant attachment: A meta-analysis on the predictive validity of the Adult Attachment Interview. Psychological Bulletin, 117, 387-403.

van Ijzendoorn, M.H., \& Bakermans-Kranenburg, M.J. (2006). DRD4 7-repeat polymorphism moderates the association between maternal unresolved loss or trauma and infant disorganization. Attachment and Human Development, 8, 291-307.

van Ijzendoorn, M.H., Schuengel, C., \& Bakermans-Kranenburg, M.K. (1999). Disorganized attachment in early childhood: Meta-analyses of precursors, concomitants and sequelae. Development and Psychopathology, 11, 225-249.

Watson, J.S. (1994). Detection of self: The perfect algorithm. En S. T. Parker, R.W. Mitchell, \& M.L. Boccia (Eds.), Self-awareness in animals and humans. New York: Cambridge University Press. 